\title{
Design of tunnel illumination control system with solar energy
}

\author{
Zhang Weijiang* \\ Tibet agriculture and Animal Husbandry university,school of electrical engineering, Linzhi, Tibet, China
}

\begin{abstract}
In order to solve the unmanned tunnel illumination in remote areas, control system is designed for tunnel lighting. Meanwhile, solar energy is used to solve the energy problem of tunnel lighting. Based on the specific requirements of the highway tunnel, the intelligent control algorithm is designed. On the basis of the algorithm, the intelligent control tunnel lighting system is realized. Control system for tunnel lighting is stable and reliable, unmanned operation can be realized. Practice shows that the intelligent tunnel lighting control system can be successfully applied in remote areas.
\end{abstract}

\section{Introduction}

The research on lighting technology of highway tunnel in foreign countries began early. After years of research and practice, the technology is relatively mature. As early as 1960s, Mont-Blanc tunnel between Italian and French has been illuminated according to the change in traffic volume. The advanced lighting control method is to ensure visual conditions. At present, the majority of the tunnel illumination system adopts the hierarchical automatic control method. Although there is a certain energy saving effect, there is still a problem of excessive lighting. The intelligent lighting control system can adjust the brightness of the tunnel in real time according to the internal and external environmental characteristics of the tunnel. While ensuring the safety of the tunnel driving, it will prevent the waste of electric energy from the excessive lighting. The intelligent lighting control system can also prolong the service life of the lamps and lanterns, and reduce the cost of management and maintenance of the tunnel. Intelligent lighting control system is another development trend of tunnel lighting design in the future.

\section{Principle of tunnel illumination}

\subsection{Installation rules of lamps and lanterns}

Layout of tunnel illumination refers to the installation position of the lamp in the tunnel wall. It could be height installation about lamp. Layout methods include: middle lamp, symmetrical bilateral lamp, bilateral interlaced lamp, etc. It depends mainly on the light of the length of the unit tunnel. The layout space of the illuminaire is mainly constrained by the light flicker and the illumination requirement. It is necessary to establish a double constraint programming model for calculating the space of lamps. The space of lamp distribution is solved with the minimum luminous flux as the objective function. In engineering design, special software could be used to determine the number of lamps and judge the space of the lamp. In order to avoid the influence of driver scintillation effect of safety and comfort. Design speed on it. The layout rules of the tunnel lamps are shown in Table 1.

Table 1. The layout rules of the tunnel lamps

\begin{tabular}{|c|c|c|}
\hline Speed $(\mathrm{km} / \mathrm{h})$ & $\begin{array}{c}\text { Twinkle } \\
\text { frequency }<2.5 \mathrm{~Hz}\end{array}$ & $\begin{array}{c}\text { Twinkle } \\
\text { frequency }>15 \mathrm{~Hz}\end{array}$ \\
\hline 100 & $>11.11$ & $<1.85$ \\
\hline 80 & $>8.88$ & $<1.48$ \\
\hline 60 & $>6.66$ & $<1.11$ \\
\hline 40 & $>4.44$ & $<0.74$ \\
\hline
\end{tabular}

\subsection{The installation height of lamps}

The determination of the installation height of the tunnel lighting fixtures is mainly influenced by the civil construction factors of the tunnel. In order to ensure the safety of tunnel traffic, the installation of equipment in the tunnel needs to be controlled within a certain limit. This limit is related to the ratio of the width and height of the tunnel. The layout of the installation of the tunnel light is shown in Figure 1.The dotted line frame in the picture is the driving limit of the vehicle. All the equipment in the tunnel should be installed outside the dotted line frame. If the height of the fixtures is very low, there may be an accident. Such accidents should be prevented the occurrence as much as possible in construction. Combined with the middle offset lamp, the lamp is installed at the middle line of the tunnel at the center line for $70 \mathrm{~cm}$ to $100 \mathrm{~cm}$. In the design and construction of tunnel lighting, the installation requirements of lamps and lanterns should be fully considered in the design and construction. At the same

\footnotetext{
*Zhang Weijiang: 1711547647@qq.com
} 
time, the errors in the field construction could not be much larger than the drawings.

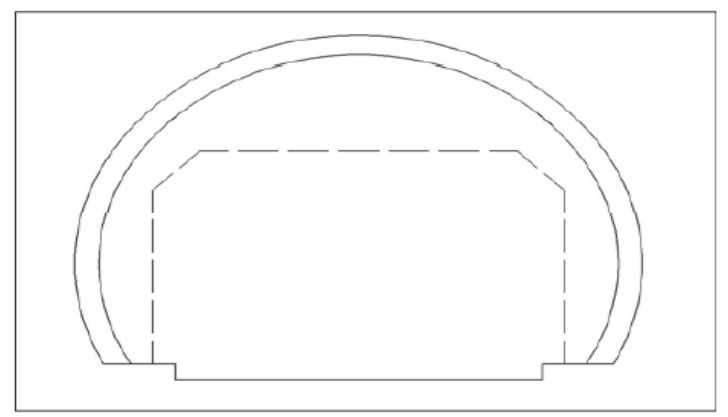

Fig. 1. The layout of the installation of the tunnel

\section{Design of control system}

\subsection{Main technical parameters and structure}

System parameters are the main basis for the design of control system. The working temperature range of the solar panel is $-20 \sim 50$. Solar panel service life is 219000 hours. The battery charge time is $2-4$ hours. Storage energy efficiency of the battery is $70 \% \sim 85 \%$. The experimental results show that the intelligent slidingmode strategy has better robust property and intelligence. The steady-state error of the whole system is not more than $3 \%$.The system can be unmanned and has some redundancy.

An intelligent control system is a system for completing some kind of control task. In a sense, intelligence is a special mapping relationship from input to output. The intelligent control system generally has 3 significant features. Known as a learning system, it is a feature of an intelligent system. A system that can be organized and coordinated for complex control tasks as a system of organizational function. The adaptability of the intelligent control system does not depend on the model of the system. The host measured the light intensity outside the tunnel through a light intensity sensor and set the value according to the first memory module. The host communicates with the slave computer in the way of RS485 communication, and the duty ratio of the output PWM regulates the output current of the LED power module. The data in the first memory module and the second memory module can be rewritten by encoding the keyboard circuit to achieve the goal of off-line adjustment of tunnel illumination and luminance. Using the LED lamp as the tunnel lamp, depending on its energy saving and reliability, it not only saves the power resources, but also has its own low power characteristics. Intelligent tunnel lighting system consists of 5 parts. The overall structure is shown in Figure 2.Solar panels convert solar energy into electrical energy which be sent into the charge and discharge controllers. Charge discharge controller can control the size of current when charging and discharging. When the discharge is required, the power of the battery is controlled by the charge and discharge controller. DC power is converted to AC 220 voltages by using inverter. The whole system has the master controller and the slave control group, that are to say, the controller is not one but more than one. The master controller communicates with the slave control through the 485 bus. The control data of the main controller can be received from the controller, and the LED can be adjusted according to the control data. In this structure, the controller adjusts the output voltage of the driver by adjusting the pulse width. In essence, the drive is equivalent to a constant current source. The control basis of the main controller mainly depends on the light intensity outside the tunnel.

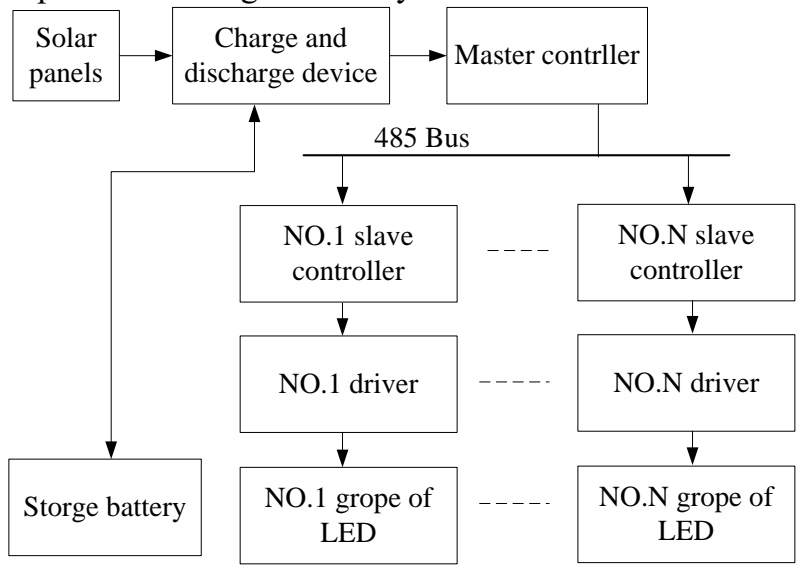

Fig. 2. The structure of control system

\subsection{The Working process of the control system}

The master controller initializes the system first and opens the total interruption, and reads the data of the light sensor. The corresponding states value of the coded keyboard is read. The corresponding result state 0 is expressed as a working mode. The master controller collects the data of the light intensity sensor. Through scale conversion, the real illumination of the current environment is obtained. By comparing with the data set from the first memory module, the pulse width adjustment value is obtained by the look-up table method. Then the pulse width adjustment data and the address data are combined into a data string and transmitted to the 485 bus through the SCI. The slave controller receives data through SCI. If there is no error in the data, the address data are compared only from address of the machine micro-controller to the address data of the master micro-controller.

The slave process data and transfer the processing results to the LED tunnel light in the form of PWM. The corresponding result state 1 is a query mode. There is only a response from the machine with the correct data format and the address, machine would correspond. Master receives data from the slave Micro-controllers through the interrupt service program. When the result corresponding to state 2 represents the rewriting state, the data from the slave Micro-controllers are sent by the host Micro-controllers. The data is written to the second memory module $10 \mathrm{AT} 24 \mathrm{C} 16$ chip. If the data of the table is changed, the output pulse width adjustment value will be changed. The corresponding state 3 represents the liaison state of the host computer. The slave micro- 
controller receives the data and displays the data to the host computer. When the result corresponds to state 4 is a system test state. Initialize the system first and open the total interruption from the master micro-controller. When the data format is judged to be wrong, enter into a wait mode. When the function code of the data format is 06 and the flag code is $5 \mathrm{~A}$, it corresponds to the working state. Master micro-controllers process the received data and the width adjustment values in the processing results to the LED drive unit, changing the illumination of the LED tunnel light. Send the current pulse width adjustment value from the 485 bus to the host microcontroller from the slave micro-controller. When the function code of the data format is 06 and the flag code is $\mathrm{AA}$, the incremental pulse width adjustment value is sent from the slave micro-controller to the LED drive unit. During the work of the control system, the flow chart of the interruption and the flow chart of the slave are shown in Figure 3, Figure 4.

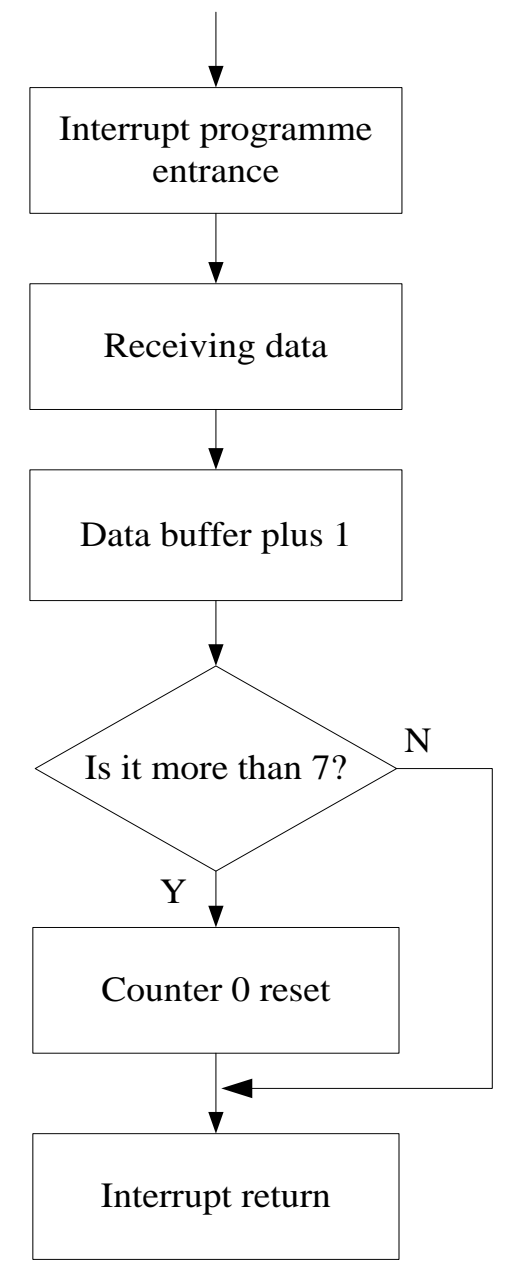

Fig. 3. Interrupt programme flow chart

The interrupt program of the system is mainly corrected when the communication is wrong. The slave control program mainly receives the signal from the host and controls the driver. At the same time, the controller needs to monitor the state of the system. The system state is divided into 3 states, namely the query state, the working state and the test state. If the system is working mode, the slave controller will output the date to the driver. If the state of the system is a query state, the output pulse width will be adjusted by the slave controller. If the state of the system is a query state, the width of the output pulse will be increased by the slave controller. The communication protocol between the master controller and the slave controller is self determined.

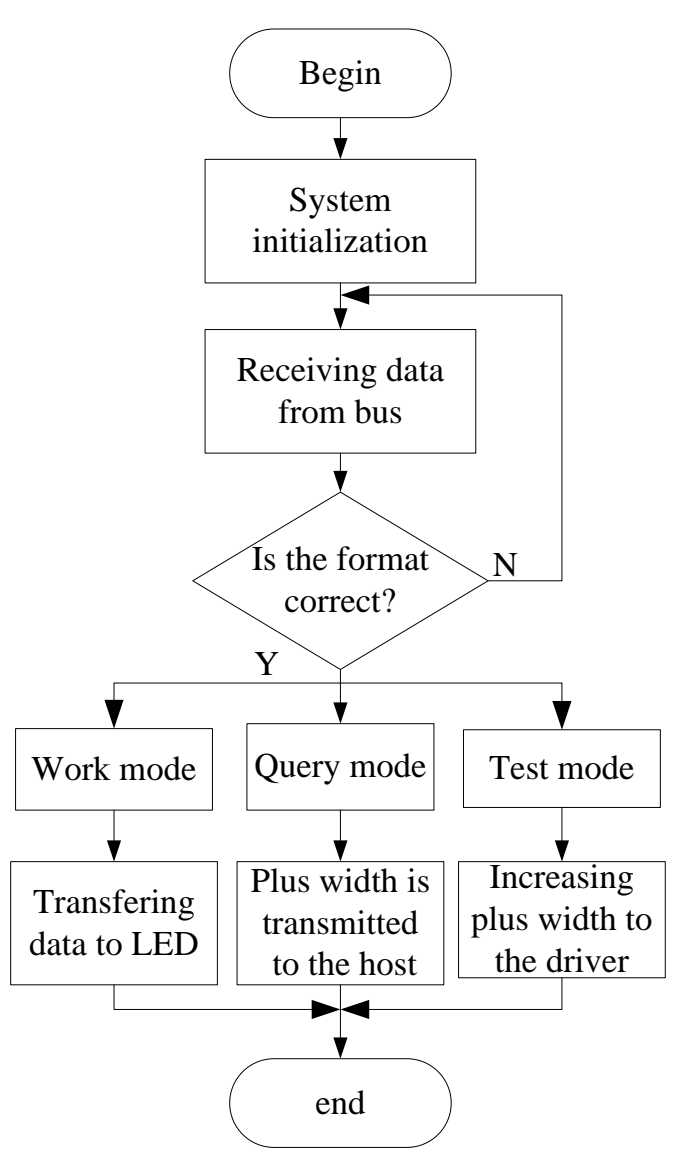

Fig. 4. The slave control programme flow chart

\section{The hardware of control system}

The coded keyboard circuit uses two 8 binary encoder CD4532B with priority to implement the hardware coding. This setting consists of a binary coded interface circuit to eliminate interference. Encoding keyboard circuit comprises a mono-stable circuit and a delay circuit, mono-stable circuit is composed of U1B, U1C, NAND gate resistor R2 and capacitor $\mathrm{C} 1$.The output ends of the input NAND U1B connection with U1C.The input terminal of $\mathrm{U} 1 \mathrm{C}$ is connected with the capacitance C1.The delay circuit is composed of gate U2D, resistor $\mathrm{R} 1$ and capacitance $\mathrm{C} 2$. The output terminal of the gate or U2D is connected to the capacitance $\mathrm{C} 1$ through resistance $\mathrm{R} 1$, and the capacitance $\mathrm{C} 1$ is connected to the ground wire. The resistance $\mathrm{R} 1$ and $\mathrm{R} 2$ are $10 \mathrm{~K}$, and the capacitance value is 0.1 . STM32 chip is used as processing chip for host micro-controllers. The main functions of the host are related to signal detection and discrimination, algorithm processing and control, and output control. The serial output signal TXD of the host micro-controller is connected to the pin 3 of the high speed and 6 N137-1.The pin 6 of the 6 N137-1 is 
connected to the pin 4 of the 485 communication special chip 6N75176. The pin 3 of the optoelectronic coupled TLP521 is connected to the pin 2 and 3 of the 6 N75176.The pin 3 of the 6 N137-2 is connected to the 6 N75176's pin 1 .The pins 6 and 7 of the 6 N75176 are connected to the 485 communication input terminal of the slave micro-controllers, $\mathrm{B}$ and A. Host microcontrollers and slave micro-controllers to receive data through the I2C bus. The display circuit uses LCD12864 for man-machine interaction and data display. The first memory module uses an AT24C16 chip as a memory chip. Pins 5 and 6 of the AT24C16 are connected to the pins 22 and 21 of the STC12c5260s2.AT24C16 pins 1, 2, $3,4,7$ grounding. The pin 8 of the AT24C16 is connected to the power VCC5. Pins 5 and 6 of the AT24C16 are connected to the power VCC5 with a $1 \mathrm{~K}$ resistance. The pin 17 of the STC12c5260s2 is connected to the base of the transistor 9012 and 901.The new power management module uses the DC power supply module TPT0505S for voltage isolation. The pin 1 of the TPT0505S is connected to the VCC5.Optical intensity sensor is used to collect external light intensity data by using BH1750FVI chip. The second memory module uses AT24C16 chip as the memory chip. The connection diagram of the communication module is shown in Figure 5.

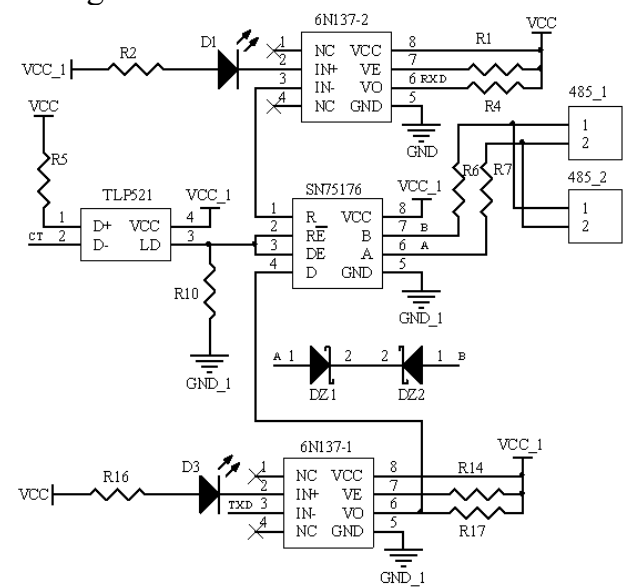

Fig. 5. The connection diagram of the communication module

All load current could not exceed the rated load current of the controller 20A.Put the controller in the position to be installed and check whether there is enough space ventilation up and down. The positive and negative pole of the battery board must be avoided installing the controller in direct sunlight, high temperature, and easy water. When the battery discharges and must switch to the use of space power to make the system work, and disconnects the inverter circuit breaker. The battery pack is made up of two batteries in series. Tunnel lighting control device can realize off-line adjustment of light intensity and brightness of tunnel lighting. The illumination intensity of the LED tunnel light can be adjusted intelligently according to the light intensity outside the tunnel. The cost of the system is low, and it can save energy and protect the environment. It has the characteristics of fast adjusting speed, low use device, low power consumption and long service life. These are obviously not enough to meet the continuous long run of the monitoring device. In addition to the light intensity, the temperature is also an important factor affecting the photoelectric conversion rate. When the temperature rises, the photoelectric conversion rate decreases obviously. In addition, the process structure of the photocell itself will be affected. The method of improving the efficiency of the panel is to cool the solar panel and change the reflection coefficient. In case of conditions, the sun can be traced to obtain greater light intensity. With the progress of materials and electronic technology, in the foreseeable future, the power resources of greater power can be obtained on a smaller area. Solar energy photovoltaic power generation will gain greater application space

The road tunnel lighting is different from the general road lighting. It is necessary to fully consider the adaptability of the human eyes and transmit the necessary visual information to the driver. When a driver enters a tunnel during the day, there will be a dark adaptation process. When driving away from the tunnel, there will be an adaptation process.

\section{Conclusions}

The tunnel lighting algorithm proposed in this paper can realize the scientific lighting of the tunnel according to the human visual principle.The master and slave control system could adjust the precision of the light.Practice shows the rationality of tunnel illumination control system in the paper.

\section{Acknowledgments}

This article has been supported by the Tibetan high-end talent introduction project in 2012. Thanks to all the colleagues and friends who have contributed to this article.

\section{References}

1. Guide for the lighting of road and underpass:CIE88 2004[S].Austria, international commission on illumination ,2004.

2. CHEN F,WANG K,QIN Z,et al.Design method of high efficient LED headlamp leans .Opics Express 2012,18(20):2096-2938.

3. He Y,Changbin L,Aiguo W,et al.LED Lighting control system in tunnel based on intelligent illumination curve $\mathrm{C} /$ /International conference on intelligent computing Technology and Automation.Berlin:IEEE,2012:698-701.

4. Parise G,Martirano L.Structured distributaion of electric system:The example of roadway tunnel archicture J.IEEE Trans.Ind.2010,46(5):2099-2015.

5. IESNA.9th:NESNA Lighting handbook. 